\title{
L'art de la communication au sein de l'équipe: ne rien laisser au hasard des interprétations
}

\author{
Anna Mascherek ${ }^{a}$, \\ Irene Kobler ${ }^{b}$, \\ Paula Bezzolac \\ a Dr phil., collaboratrice \\ scientifique, sécurité des \\ patients suisse \\ b Gestionnaire de projet, \\ sécurité des patients suisse \\ c Directrice adjointe, \\ sécurité des patients suisse
}

Correspondance:

Paula Bezzola, MPH

sécurité des patients suisse

Asylstrasse 77

CH-8032 Zurich

Tél. 0432441480

Fax 0432441481

bezzola[at]patientensicherheit.ch
La communication a une influence décisive sur le comportement des membres d'une équipe: il ne s'agit pas seulement de faire passer un message, mais de savoir qui dit quoi, quand et comment. Correctement appliquée, la check-list chirurgicale (check-list de l'OMS) soutient et facilite une communication structurée entre les intervenants. Ces échanges aident l'équipe à acquérir un modèle mental commun de la situation et permettent de reconnaître à temps les risques pour la sécurité. Communiquer de manière claire et concise ne s'improvise pas: c'est un art à exercer.

\section{Avons-nous la même conception et la même perception des choses?}

Lorsque nous échangeons avec d'autres personnes, nous pensons souvent que nous nous exprimons de façon claire et sommes persuadés que notre interlocuteur a reçu l'information telle que nous la voyons. Cette croyance est d'autant plus forte que nous connaissons bien le contexte (= salle d'opération), la situation (= intervention de routine sur un patient) et/ou les intervenants (= personnel médical de différents groupes professionnels). Le fait d'œuvrer dans un cadre commun et de se concentrer sur une même tâche porte à croire que toutes les personnes présentes ont la même vision et qu'il existe un modèle mental unique. Cette concordance apparente masque les différences de perspective au sein de l'équipe et empêche de prendre conscience des divergences et de leurs conséquences. Des études ont montré que des personnes travaillant ensemble pouvaient avoir des conceptions très différentes et que celles-ci représentaient un risque potentiel pour la sécurité. Sexton [1] a notamment demandé à du personnel médical de se prononcer sur l'affirmation suivante: «Même si je suis fatigué(e), je reste efficace et performant(e) dans des situations critiques». Si cette déclaration a été approuvée par 60\% des professionnels interrogés, seuls $47 \%$ des anesthésistes y adhèrent, contre $70 \%$ des chirurgiens. Cette question simple, mais non moins déterminante, illustre de façon exemplaire comment les avis peuvent diverger et conduire des personnes à interpréter différemment une situation.

\section{Les différences de perception, un risque pour la sécurité}

La standardisation des processus, la qualité de la formation des professionnels et l'investissement per- sonnel de chacun font que les différences de perception majeures sont certes rares entre les membres de l'équipe chirurgicale et que les divergences mineures dans la façon de concevoir une situation restent la plupart du temps sans conséquence. Mais dans certains cas, des erreurs se produisent suite à des malentendus liés précisément à un manque de communication [2]. En l'absence d'échanges structurés permettant à l'équipe de se forger une compréhension commune de la situation avant l'opération, chacun s'en tient à sa perception des choses, ce qui génère un risque d'erreurs dues à une mauvaise interprétation ou à un déficit d'information.

Par exemple, un orthopédiste ne saurait tenir pour acquis que les implants spéciaux nécessaires pour une intervention seront automatiquement disponibles le moment venu. Il se peut en effet que l'on ait oublié les délais de commande, que les implants

\section{«Une même situation peut donner lieu à des appréciations très diverses de la part des groupes professionnels.»}

soient utilisés ailleurs ou que l'on imagine, à tort, que les responsables de la gestion s'en sont occupés de leur propre chef. Si ce point n'est pas abordé explicitement avant l'intervention, celle-ci pourrait devoir être retardée ou stoppée jusqu'à ce que le matériel manquant puisse être obtenu, par exemple auprès d'un autre hôpital, et ce alors que le patient est déjà sous anesthésie ou que l'opération a déjà débuté. Les erreurs de cet ordre peuvent et doivent être évitées. Une bonne communication au sein de l'équipe permet de contribuer efficacement à cette prévention. Aujourd'hui encore, ce point n'est pourtant pas une évidence en salle d'opération, comme l'atteste une étude de Makary et al. [3] réalisée auprès d'anesthésistes, de chirurgiens et de personnel infirmier. Les résultats montrent que les participants ont une appréciation fort différente de la qualité de la communication au quotidien: si les chirurgiens estiment à 87\% que la communication avec le personnel infirmier est bonne ou très bonne, seuls $48 \%$ dudit personnel partagent cet avis (pour une situation 
identique). De même, $84 \%$ des chirurgiens ont jugé que la communication avec les anesthésistes était bonne ou très bonne, alors que ces derniers ne sont que 70\% à exprimer cette opinion. Une même situation peut donc donner lieu à des appréciations très diverses de la part des groupes professionnels - autant de divergences qui peuvent être une source d'erreurs.

\section{La communication structurée aide à éviter les erreurs}

La manière dont les membres d'une équipe communiquent entre eux est un facteur déterminant dans la

Figure 1

Communication bilatérale et communication structurée, en boucle fermée.

Communication bilatérale
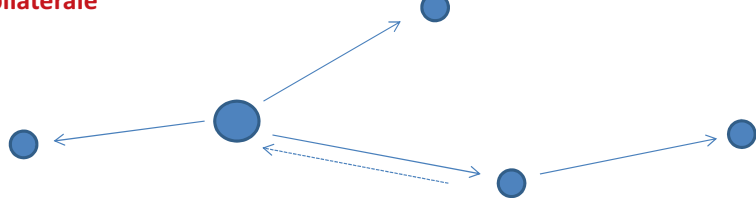

Communication structurée, en boucle fermée

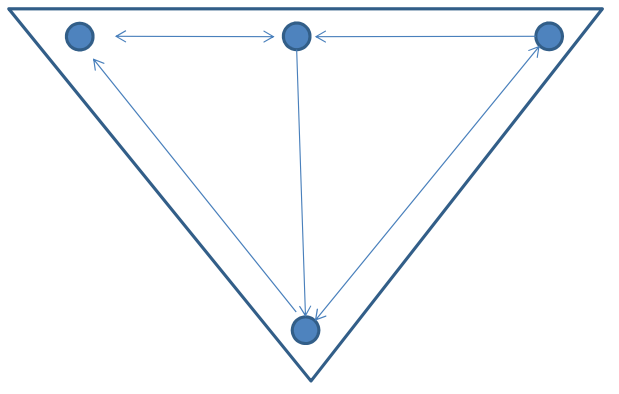

O sécurité des patients suisse

Figure 2

Une communication en trois phases. La communication en boucle fermée se déroule en trois phases.

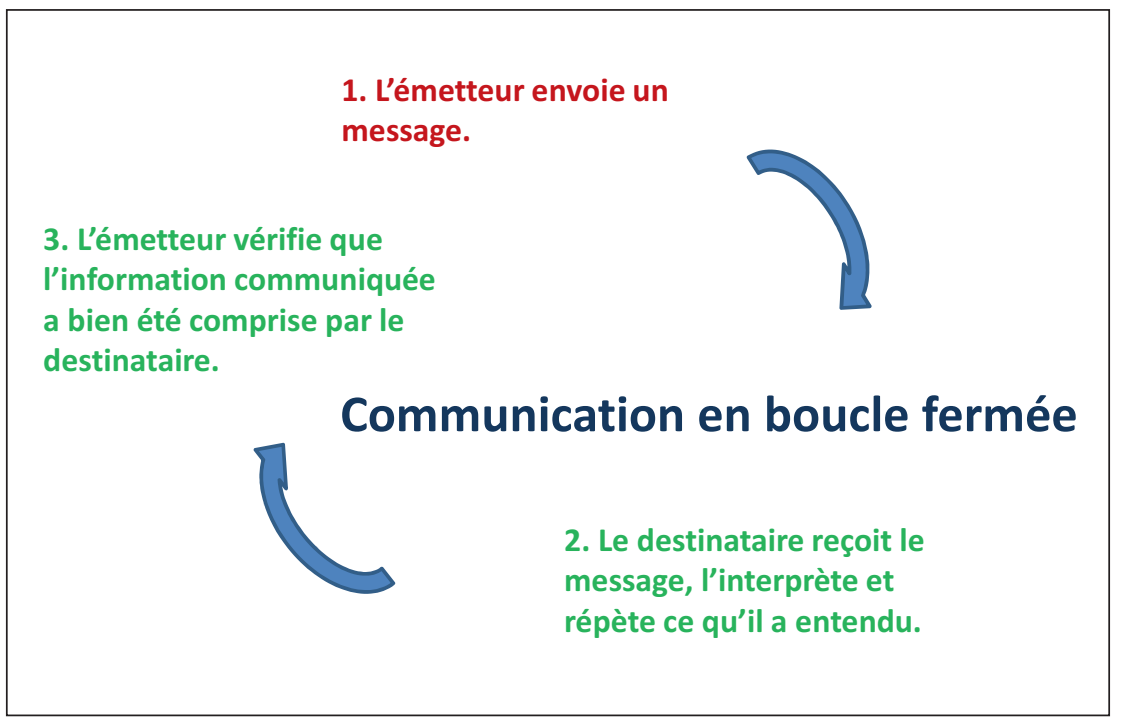

prévention des interprétations erronées et des malentendus. Plus les échanges sont standardisés et structurés, plus il y a de chances que soient transmises toutes les informations nécessaires à une bonne compréhension de la situation. Une communication structurée aide l'équipe à acquérir une vision commune et encourage chacun à s'exprimer en cas d'incertitude.

\section{Qu'est-ce qui caractérise une communication structurée?}

Une communication structurée doit être objective, c'est-à-dire neutre, claire, précise, complète et concise. Elle présente également les caractéristiques suivantes:

- les points à traiter et la manière de les aborder sont définis à l'avance;

- tous les membres de l'équipe écoutent;

- chacun participe aux échanges selon le rôle ou la fonction défini(e) dans le déroulement structuré;

- tous osent exprimer leurs incertitudes (Speak up);

- les informations sont répétées et validées (closed loop communication $=$ communication en boucle fermée).

Souvent, il y a bien échange d'informations durant le processus, mais celles-ci sont communiquées de manière bilatérale et non organisée. La communication structurée vise à s'assurer que tous disposent des données nécessaires et sont en mesure de détecter les éventuels manquements. Le fait de reconfirmer systématiquement les informations échangées aide en outre à éviter les malentendus. La communication en boucle fermée est particulièrement importante pour les vérifications de sécurité.

\section{Une communication structurée est-elle réellement synonyme de sécurité accrue?}

Mettre en place une communication structurée tout en assurant une utilisation correcte de la check-list représente un investissement non négligeable. Il est donc légitime de se demander si la qualité de la communication au sein de l'équipe a des effets tangibles et mesurables sur la sécurité des patients ou reste un simple «facteur de bien-être». Neily et al. [4] ont voulu savoir si ce paramètre avait une influence sur le taux de mortalité. A cet effet, plusieurs équipes chirurgicales dans leur composition originelle (chirurgien, anesthésiste, infirmière anesthésiste, équipe technique et infirmière) ont suivi un entraînement à la communication. Les résultats indiquent un taux de mortalité sensiblement plus faible pour les patients du groupe ayant bénéficié de cet entraînement intensif et attestent que les effets positifs sont liés à des changements au niveau de la communication. Cette étude démontre qu'une bonne communication interpersonnelle en salle d'opération ne fait pas qu'améliorer le travail quotidien de l'équipe, 
mais a aussi des répercussions directes sur le résultat du traitement et, partant, sur la sécurité du patient.

\section{La check-list chirurgicale, une aide efficace à la communication}

Lorsqu'elle est employée de manière correcte et systématique, la check-list chirurgicale (check-list de l'OMS) est un instrument éprouvé et efficace aidant les membres de l'équipe à échanger de façon structurée, standardisée et objective les informations essentielles concernant l'opération prévue. En peu de

\section{«La communication structurée vise à s'assurer que tous disposent des données nécessaires et sont en mesure de détecter les éventuels manquements.»}

\section{Sujets \\ d'actualité \\ du forum \\ Venez débattre avec nous! Dans la rubrique forum, nous présentons régulière- ment des sujets d'actua- lité politique, économique et scientifique ayant trait au système de santé. Donnez votre avis ou commentez les affirmations de vos confrères. Pour accéder au forum: www.bullmed.ch/forum/}

temps - il faut en moyenne moins d'une minute pour la passer en revue -, elle permet à l'ensemble des participants de parvenir à une compréhension commune de la situation en abordant et en clarifiant tous les aspects importants (5).

\section{La sécurité passe par la communication}

Parallèlement à l'intégration systématique de la check-list, la communication au sein de l'équipe constitue, pour toutes les raisons évoquées ci-dessus, un élément essentiel du programme pilote national «progress! La sécurité en chirurgie» (voir encadré). Une culture empreinte de respect et d'estime permet de soutenir une communication factuelle et les échanges standardisés et structurés contribuent à leur tour à créer un cadre professionnel et objectif facilitant la prise de parole.

Pour que la check-list puisse déployer ses effets positifs sur la communication au sein de l'équipe, il est essentiel d'adapter cet instrument à l'établissement en y associant le personnel concerné, puis de le tester et de s'entraîner à l'utiliser. Il convient en particulier de définir à l'avance la manière de procéder et les formules à employer, qui devront être claires pour tous, et de prévoir des normes et des règles qui seront appliquées de façon complète et systématique. Les cadres assument à cet égard un rôle de modèle important.

\section{Références}

1 Sexton JB, Thomas EJ, Helmreich RL. Error, stress, and teamwork in medicine and aviation: cross sectional surveys. BMJ. 2000;320(7237):745-9.

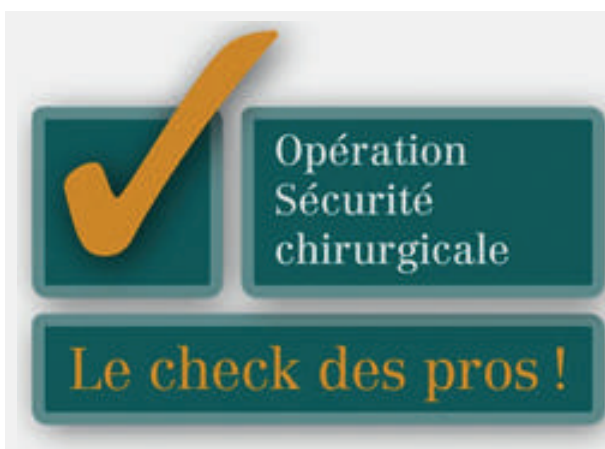

Programme pilote national progress!

Les bases du programme pilote national progress! réalisé par "sécurité des patients suisse" ont été élaborées en étroite collaboration avec de nombreux spécialistes suisses du domaine de la santé, des sociétés membres de la $\mathrm{fmCh}$ ainsi que des associations professionnelles du domaine opératoire et des infirmiers et infirmières anesthésistes. L'objectif consiste à assurer une utilisation correcte et complète de la checklist chirurgicale pour tous les patients (observance de $100 \%$ ). L'accent est mis notamment sur l'amélioration de la communication au sein de l'équipe, qui représente un facteur essentiel. Entre 2013 et 2015, dans le cadre d'un projet d'approfondissement de deux ans, dix hôpitaux pilotes mènent plusieurs enquêtes et activités prédéfinies au sein de groupes de projet interprofessionnels et partagent leurs expériences lors de quatre ateliers communs.

Pour en savoir plus: www.securitedespatients.ch, Paula Bezzola, MPH, responsable du programme pilote progress! et directrice adjointe, tél. 043 2441480 , bezzola[at]patientensicherheit.ch

2 Gawande AA, Zinner MJ, Studdert DM, Brennan TA. Analysis of errors reported by surgeons at three teaching hospitals. Surgery. 2003;133(6):614-21.

3 Makary MA, Sexton JB, Freischlag J, Holzmueller C, Millman EA, Rowen L, et al. Operating room teamwork among physicians and nurses: teamwork in the eye of the beholder. J Am Coll Surg. 2006;202:746-52.

4 Neily J, Mills PD, Young-Xu Y, Carney BT, West P, Berger DH, et al. Association Between Implementation of a Medical Team Training Program and Surgical Mortality. JAMA. 2010;304(15):1693-700.

5 Bezzola P, Hochreutener MA, Schwappach DLB. Opération Sécurité chirurgicale. La liste de contrôle chirurgicale et sa mise en œuvre: culture-équipe-instrument. Zurich: Fondation pour la Sécurité des Patients; 2012. 